\title{
Time Series Modelling of Inflation in Botswana Using Monthly Consumer Price Indices
}

\author{
Kesaobaka Molebatsi ${ }^{1} \&$ Mpho Raboloko $^{2}$ \\ ${ }^{1}$ Department of Statistics, University of Botswana, Gaborone, Botswana \\ ${ }^{2}$ Botswana Institute for Development Policy Analysis, Gaborone, Botswana \\ Correspondence: Kesaobaka Molebatsi, Department of Statistics, University of Botswana, Gaborone, Botswana. \\ E-mail: kesaobaka.molebatsi@ mopipi.ub.bw
}

Received: December 15, 2015

Accepted: January 13, 2016

Online Published: February 25, 2016

doi:10.5539/ijef.v8n3p15

URL: http://dx.doi.org/10.5539/ijef.v8n3p15

\begin{abstract}
This paper identifies an autoregressive integrated moving average (ARIMA $(1,1,1)$ ) model that can be used to model inflation measured by the consumer price index (CPI) for Botswana. The paper proceeds to improve the model by incorporating the generalized autoregressive conditional heteroscedasticity (ARCH/GARCH) model that takes into consideration volatility in the series. Ultimately, CPI is forecast using the two models, ARIMA (1, $1,1)$ and $\operatorname{ARIMA}(1,1,1)+\operatorname{GARCH}(1,2)$ and compared with the actual CPI. Both models perform well in terms of forecasting as their 95 percent confidence intervals cover the actual CPI. Marginal differences that favour the inclusion of the ARCH/GARCH components were observed when testing for normality among error terms. The paper also reveals that volatility for Botswana's CPI is low as shown by small values of ARCH/GARCH components.
\end{abstract}

Keywords: ARIMA, ARCH, GARCH, CPI, inflation

\section{Introduction}

The primary objective of monetary policy, both in Botswana and elsewhere is to maintain low and stable rates of inflation. A low and stable inflation is crucial in maintaining a stable macroeconomic environment. One of the major economic challenges facing developing countries is rising levels of inflation. Higher rates of inflation cause uncertainty about future inflation by distorting the price mechanism. Inflation affects an economy by way of minimizing the purchasing power of economic players. Consequently, inflation reduces the purchasing power per unit of currency and a loss of real value in the medium of exchange and unit of account in the economy. Inflation is therefore, described as a major focus of economic policy worldwide (David, 2001). It is in this context that the primary objective of Bank of Botswana's monetary policy is to achieve price stability, which is defined as a sustainable level of inflation that is within the medium-term objective range of 3-6 percent.

The annual inflation rate fell below the Bank of Botswana's medium-term objective range of 3-6 percent in February 2015. The decline in the inflation rate is attributed to the fall in global commodity prices. Despite the fall in inflation in the last couple of years, the Central Bank is still concerned about inflation. Historic rates of inflation suggest that inflation has always been a concern for Botswana. The inflation rate has consistently overshot the upper band of the objective range since 2007. From January to December 2011, the inflation rate averaged at 8.5 percent (African Economic Outlook, 2012). Monetary policy is therefore faced with the challenge of maintaining low and stable prices as one of the key macroeconomic objectives for Botswana. In view of that, we note the importance of investigating the trends of inflation in order to inform policy with regard to keeping inflation low and stable.

\section{Literature Review}

To the best of our knowledge, there are few studies on time series modelling of Botswana's inflation and none of these studies factor in the volatility concept. Atta, Jefferis, and Mannathoko (1995) examined Botswana's price and inflation relationships and their interaction through cointegration analysis and dynamic error correction models, in order to establish the link between long run equilibrium prices and short run inflation. They found that exchange rate and South African prices rather than money supply was cointegrated with prices.

Taye (2013) investigated the determinants of inflation in Botswana and Bank of Botswana's medium-term 
objective range. The author fitted the auto regressive distributed lag (ARDL) model on the quarterly growth rates of the Botswana consumer price index (CPIB) data. The study found that price inertia, real GDP, money supply and South African prices played a dominant role in determining inflation in Botswana. The study also revealed that the probability that the Bank of Botswana will achieve its medium-term objective range of 3 to 6 per cent in the medium-term was very low. Even though the ARDL model is advantageous due to its ability to deal with long-run relationships and the short-run dynamics of the variables, it assumes that the error terms are serially uncorrelated with zero means and constant variance-covariance which may not be true all the time (Taye, 2013)

Alnaa and Ahiakpor (2011) used ARIMA procedure to model inflation in Ghana over the years 2000 to 2010. The study revealed that inflation followed ARIMA $(6,1,6)$ order. Even though the model built proved to be efficient with volatility estimated at 4.291 (which was termed moderate), the study failed to incorporate the volatility aspect into the model. We argue that volatility can be modelled by improving the ARIMA model with the ARCH model for Botswana's monthly inflation.

Most economic and financial series assume a non-constant conditional variance (heteroscedastic) that often comes when some new information is available, and inflation is not an exception. This is in contrast to the traditional time series models which have a constant conditional variance. For this reason, the traditional time series models do not perform well when used to forecast these kinds of series (Nortey, Mbeah-Baiden, Dasah and Mettle, 2014). It has become a standard procedure to estimate a forecasting equation of inflation, from which the conditional variance of forecast errors is extracted and used as a measure of uncertainty (Ma, 1998). In view of this, ARCH models of inflation are well suited for deriving inflation uncertainty. The model assumes that inflation uncertainty (the conditional variance of inflation) depends on the size of past squared errors in forecasting inflation. The ARCH model was introduced by Engle (1982) and modified by Bollerslev (1986) to a more generalized form, the GARCH model. The GARCH model has been used most widely as a variant of the ARCH model. The GARCH model imposes restrictions on the parameters to assure positive variances.

\section{Methodology}

\subsection{ARIMA Model}

ARIMA is a forecasting technique that projects the future values of a series based entirely on its own inertia. ARIMA models are well known for being simple, robust, and parsimonious, and for providing good results (Valle, 2002). ARIMA methodology attempts to describe the movements in a stationary time series as a function of autoregressive (AR) and moving average (MA) parameters.

The ARIMA (p, d, q) model is defined as follows:

$$
\phi_{p}(B)(1-B)^{d} Z_{t}=\theta_{0}+\theta_{q}(B) \alpha_{t},
$$

where the stationary AR operator $\phi_{p}(B)=\left(1-\phi_{1} B-\ldots-\phi_{p} B^{P}\right)$ and the invertible MA operator $\theta_{q}(B)=\left(1-\theta_{1} B-\ldots-\theta_{q} B^{q}\right)$ share no common factors. The parameter $\theta_{0}$ plays very different roles for $\mathrm{d}=0$ and $\mathrm{d}>0$. When $\mathrm{d}=0$, the original process is stationary, $\theta_{0}$ is related to the mean of the process, that is, $\theta_{0}=\mu\left(1-\phi_{1}-\ldots-\phi_{p}\right)$. When $d \geq 0$, however, $\phi_{1}$ is called the deterministic trend term and is often omitted from the model (Wei, 2006).

\subsection{ARCH and GARCH Models}

One of the main assumptions of the standard regression analysis and regression models with autocorrelated errors is that the variance $\delta^{2}$, of error terms $n_{t}$ is constant. However, in many practical applications, this assumption may not be realistic. Wei (2006) proposed models that incorporate the possibility of non-constant error variance and called them heteroscedasticity models. The structure of the Autoregressive Conditional Heteroscedasticity $(\mathrm{ARCH})$ model, the one that takes into consideration a varying variance is given as follows:

$$
Y_{t}=X_{t}{ }_{t} \beta+\varepsilon_{t}
$$

Where $\varepsilon_{t}=n_{t}$ and the $n_{t}$ are uncorrelated but have variances that change over time. Following Engle (1982), we assume that the error term can be modelled as:

$$
n_{t}=\sigma_{t} e_{t}
$$


where the $e_{t}$ are i.i.d. random variables with mean 0 and variance 1 , independent of past realisations of $n_{t-i}$ and

$$
\sigma_{t}^{2}=\theta_{0}+\theta_{1} n_{t-1}^{2}+\ldots+\theta_{2} n_{t-2}^{2}+\ldots+\theta_{s} n_{t-s}^{2}
$$

Given all the information up to time (t-1), the conditional variance of the $n_{t}$ becomes

$$
\begin{aligned}
& \operatorname{Var}_{t-1}\left(n_{t}\right)=E_{t-1}\left(n_{t}^{2}\right)=E\left(n_{t}^{2} \mid n_{t-1}, n_{t-2}, \ldots\right)=\sigma_{t}^{2} \\
& =\theta_{0}+\theta_{1} n_{t-1}^{2}+\ldots+\theta_{s} n_{t-s}^{2}
\end{aligned}
$$

Which is related to the squares of past errors, and it changes over time. Equation 4 is simply the optimal forecast of $n_{t}^{2}$ if $n_{t}^{2}$ follows the following AR (S) model:

$$
n_{t}^{2}=\theta_{0}+\theta_{1} n_{t-1}^{2}+\theta_{2} n_{t-2}^{2}+\ldots+\theta_{s} n_{t-s}^{2}+\alpha_{t}
$$

where $\alpha_{t}$ is an $\mathrm{N}\left(0, \sigma_{t}^{2}\right)$ white noise process. Hence Engle (1982) called the model of the error term $n_{t}$ with the variance specification given in (3) and (4) the autoregressive conditional heteroscedasticity (ARCH) model and denoted by ARCH (s).

A natural extension to the ARCH model is to consider that the conditional variance of the error process is related not only to the squares of past errors but also to the past conditional variances. Thus we have the more general error process.

$$
n_{t}=\sigma_{t} e_{t}
$$

Where the $e_{t}$ are i.i.d. random variables with mean 0 and variance 1 , independent of past realisations of $n_{t-i}$.

$$
\sigma_{t}^{2}=\theta_{0}+\phi_{1} \sigma_{t-1}^{2}+\ldots+\phi_{r} \sigma_{t-r}^{2}+\theta_{1} n_{t-1}^{2}+\ldots+\theta_{s} n_{t-s}^{2}
$$

And the roots of $\left(1-\phi_{1} B-\ldots-\phi_{r} B^{r}\right)=0$ are outside the unit circle. To guarantee $\sigma_{t}^{2}>0$, we assume that $\theta_{0}>0$ and that $\phi_{i}$ and $\theta_{j}$ are nonnegative. The model for the error term in (8) is called the generalized autoregressive conditional heteroscedasticity $(\mathrm{GARCH})$ model of order $(\mathrm{r}, \mathrm{s})$ and is denoted by GARCH $(\mathrm{r}, \mathrm{s})$. Clearly when $\mathrm{r}=0$, the model reduces to $\mathrm{ARCH}$ (s) process.

We use the Akaike Information Criterion (AIC) to choose a better model. The AIC statistic is discussed below:

$$
A I C=\ln \frac{\sum_{i=1}^{T} \hat{\varepsilon}_{i}^{2}}{T-n}+\frac{2 n}{T}
$$

where $\hat{\varepsilon}_{i}^{2}$ is the estimated squared residuals of the model, $\mathrm{T}$ is the number of observations in the sample and $\mathrm{n}$ is the number parameters estimated including the constant. The procedure dictates that the model with the smallest AIC statistic is the better hence should be selected.

\section{Results and Discussions}

Botswana consumer price index records obtained from Bank of Botswana reports from January 2005 to December 2014 are used for this paper and the 2015 data is used to evaluate the precision of the models. The descriptive statistics of the series are presented in Table 1. The maximum and minimum recorded were 15.1 (August 2008) and 3.8 (December 2014) with mean and standard deviation of 8.13 and 2.77 respectively. 
Table 1. Descriptive statistics of monthly consumer price index

\begin{tabular}{lccc}
\hline Minimum & Mean & Maximum & Std Dev \\
\hline 3.800 & 8.13 & 15.1 & 2.77 \\
\hline
\end{tabular}

It is evident from Appendix A that neither the original series nor its log transformed is stationary, hence differencing is necessary. The Augmented Dickey Fuller test (ADF) was also used to check for the stationarity constraint. The test is based on the assumption that the series is a random walk and hence not stationary, and was conducted without a constant, with a constant and with a constant and trend. The results revealed that Botswana's monthly consumer price index was not stationary until at lag 4 with a p-value of 0.01 . However, after differencing once, the series became stationary. The partial autocorrelation, autocorrelation functions as well as Akaike Information Criterion (AIC) are used to select the level of differencing, autocorrelation and moving average in order to meet the stationarity assumption and parsimony. These methods yielded ARIMA (1, $1,1)$ on the original series (not the log) with AIC of 242.1900: that is, the series was differenced once, and orders (1) of both autocorrelation and moving average components were selected.

Next we use the maximum likelihood method to estimate the parameters. The autocorrelation and moving average parameters, their standard errors, corresponding p-values as well as other statistics are presented in Table 2. We note that all the two parameters are significant at 5 per cent level of confidence. The p-value for Ljung-Box statistic is 0.7511 which implies that the null hypothesis of no autocorrelation is accepted after lag one, thereby implying that the model fitted is appropriate.

Table 2. Parameters, standard errors and p-values for ARIMA $(1,1,1)$

\begin{tabular}{lll}
\hline & $\operatorname{Ar}(1)$ & Ma (1) \\
\hline Coefficient & 0.7105 & -0.4048 \\
Standard Error & 0.1408 & 0.1813 \\
P-value & $\mathrm{P}<0.0001$ & 0.0256 \\
\hline
\end{tabular}

Sigma^2 $^{\wedge}$ 0.4254, log likelihood $=-118.09$, AIC=242.19, P-value for Ljung-Box $=0.7511$.

Before forecasting the CPI for January 2015, we improve the ARIMA model by incorporating into the analysis a class of volatility models for the residuals due to the reasons discussed earlier. We therefore attempt to fit the ARCH/GARCH models to the error terms of the fitted ARIMA model and compare the two. The ARCH/GARCH models use the squared error terms of the ARIMA model and the results are reported. These values are plotted in Appendix B and they depict clusters of volatility as well as some significant lags hence a need for ARCH/GARCH components. The method entails comparing the AIC statistics obtained from different models of the same kind and selecting the one that yields the minimum. According to this criterion, a GARCH (1, 2 ) is selected with AIC of 228.0132.

Table 3. Parameters and their standard errors for $\operatorname{GARCH}(1,2) \mathrm{P}$-value for Ljung-Box $=0.7021$

\begin{tabular}{lllll}
\hline & Constant & $\mathrm{A}(1)$ & $\mathrm{A}(2)$ & $\mathrm{B}(1)$ \\
\hline Coefficient & 0.0726 & 0.07980 & 1.0264 & 0.2667 \\
Standard Error & 0.0141 & 0.0988 & 0.1585 & 0.0689 \\
P-value & $\mathrm{P}<0.0001$ & 0.3232 & $\mathrm{P}<0.0001$ & 0.0001 \\
\hline
\end{tabular}

Table 3 gives the GARCH coefficients, standard errors and their corresponding Ljung-Box p-values for the model. We observe that the p-values of all parameters except the second are less than 0.05 , indicating that they are significant. In addition, p-value for Ljung-Box is greater than 0.05 , and hence we accept the null hypothesis that autocorrelation among residuals is absent after lagging error terms. The model thus represents the residuals well. Next we compare the results from ARIMA model and the combined ARIMA-ARCH/GARCH model.

Table 4. 1-Step ahead forecast values and $95 \%$ CI for the two models

\begin{tabular}{lcccc}
\hline & Forecast & Lower & Upper & Actual \\
\hline Model & & & & \\
ARIMA $(1,1,1)$ & 3.6357 & 2.3574 & 4.9140 & 3.60 \\
Full model of ARIMA $(1,1,1)+\operatorname{GARCH}(1,2)$ & 3.7037 & 2.4254 & 4.9820 & \\
\hline
\end{tabular}


The forecasted values for January 2015 by the two models being ARIMA $(1,1,1)$ and ARIMA $(1,1,1)+$ $\operatorname{GARCH}(1,2)$ are 3.64 and 3.70 respectively, which are close to the 3.60 recorded by the Bank of Botswana for the same period even though the ARIMA was more closer. Even though there were slight differences, all the three actual values fell within the 95 per cent confidence intervals for the two models, implying that the models are appropriate for the consumer price index. Appendix $\mathrm{C}$ depicts the conditional variances that reflect the volatility somewhere in the middle of the series; this corresponds to April 2008 to December 2009 and matches with the period where the CPI reduced significantly from more than 14 to less than 10 . We also compare the Q-Q plots of residuals of the two models which are plotted as Appendix D. We observe a very marginal difference that favours the ARIMA-GARCH model in terms of the normality assumption.

\section{Conclusions}

The analysis suggests that ARIMA models can be used to model Botswana consumer price index. The series followed ARIMA $(1,1,1)$. This class of models does not take into consideration the volatility that often comes when some new information is available, hence can be improved by the ARCH/GARCH class that bridges this gap and allows for heteroscedasticity of error terms. However, it is evident that Botswana's consumer price index is weak in volatility as depicted by low levels of the ARCH/GARCH components. This is also evidenced by the ARIMA estimates and Q-Q plots that are close to ARCH/GARCH estimates. The findings imply that over the achievement of keeping the prices within the medium-term target, Botswana has also managed to keep consumer price index stable. The actual one step ahead values fell within the 95 per cent confidence intervals for the two model predictions. These models should be fit on stationary time series hence the data has to be first transformed before further modelling.

\section{References}

Abdus, S. M., Salam, S., \& Feridun, M. (2007). Modeling and Forecasting Pakistan's Inflation by Using Time Series ARIMA Models.

African Economic Outlook. (2012). Botswana.

Alnaa, S., \& Ahiakpor, F. (2011). ARIMA (autoregressive integrated moving average) approach to predicting inflation in Ghana. Journal of Economics and International Finance, 4(3), 83-87.

Amos, C. (2010). Time series modelling with application to South African inflation data. M.S. Thesis, University of Kwazulu Natal Kwazulu Natal.

Atta, J. K., Jefferis, K. R., \& Mannathoko, I. (1996). Small Country Experiences with Exchange Rates and Inflation: The Case of Botswana. Journal of African Economies. http://dx.doi.org/10.1093/oxfordjournals.jae.a020906

Bank of Botswana. (2014). Mid-Term Review of The 2014 Monetary Policy Statement.

Bollerslev, T. (1986). Generalized Autoregressive Conditional Heteroskedasticity. Journal of Econometrics, 31, 307-327. http://dx.doi.org/10.1016/0304-4076(86)90063-1

David, F. H. (2001). Modeling UK inflation, 1875-1991. J. Appl. Econom., 16(3), 255-275. http://dx.doi.org/10.1002/jae.615

Engle, R. F. (1982). Autoregressive Conditional Heteroscedasticity with Estimates of the Variance of United Kingdom Inflation. Econometrica, 50, 987-1007. http://dx.doi.org/10.2307/1912773

Ma, H. (1998). Inflation, Uncertainty and Growth in Columbia. IMF working Paper, WP/98/161.

Nortey, E. N. N., Mbeah-Baiden, B., Dasah, J. B., \& Mettle, F. O. (2014). Modelling Rates of Inflation in Ghana: An Application of ARCH Models.

Svenson, L. E. O. (2007). Inflation Targeting, Princeton University. CEPS Working Paper No. 144.

Taye, H. K. (2013). Inflation Dynamics in Botswana and Bank of Botswana's Medium-Term Objective Range. BIDPA Working Paper 36.

Valle, H. A. (2002). Inflation Forecsts with AIMA and Vector Autoregressive Models in Guatemala. Working Paper 1.

Wei, W. W. S. (2006). Time Series Analysis: Univariate and Multivariate Methods, Greg Tobin (2nd ed.). 


\section{Appendix A}
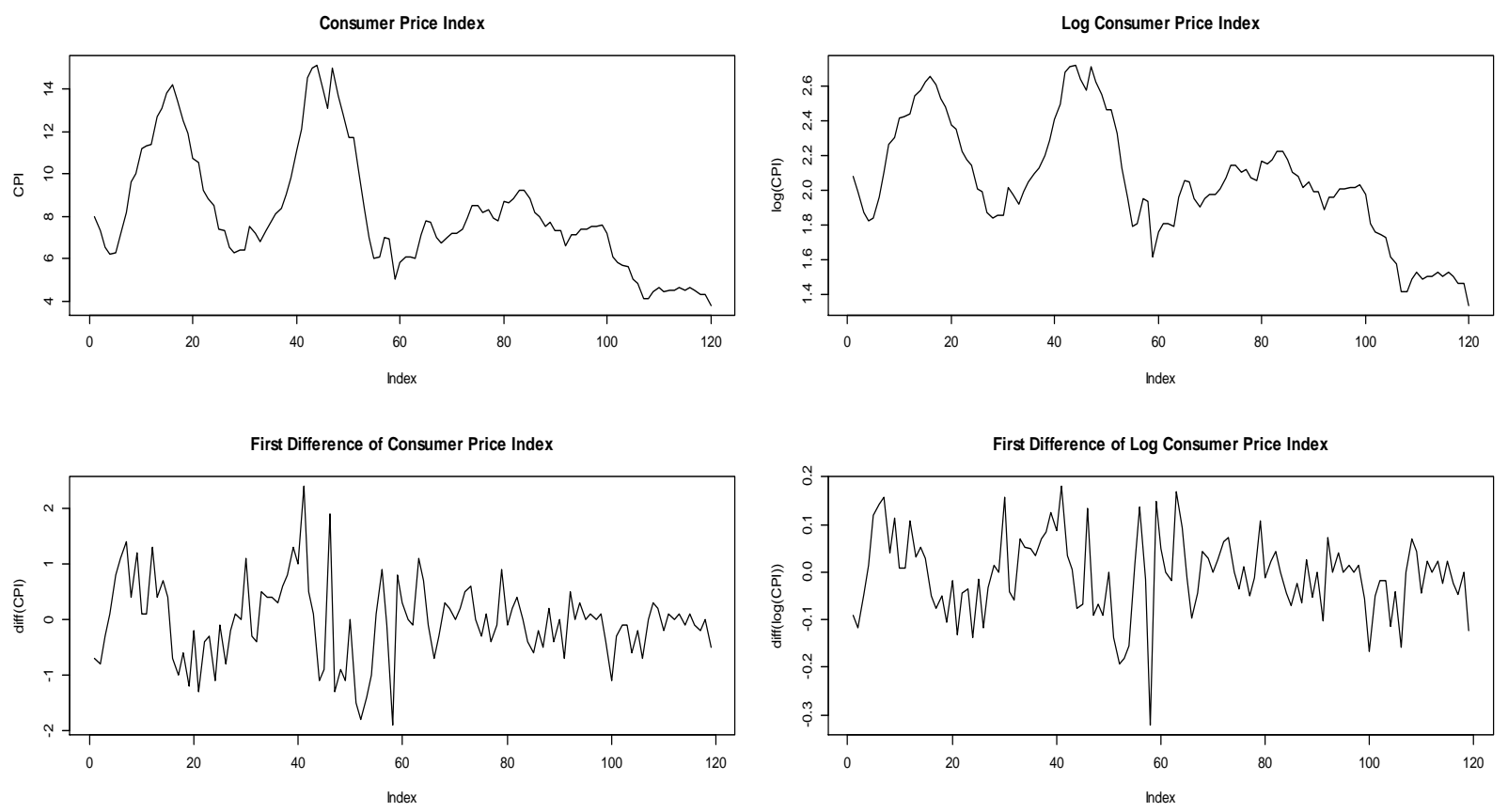

Second Difference of Consumer Price Index

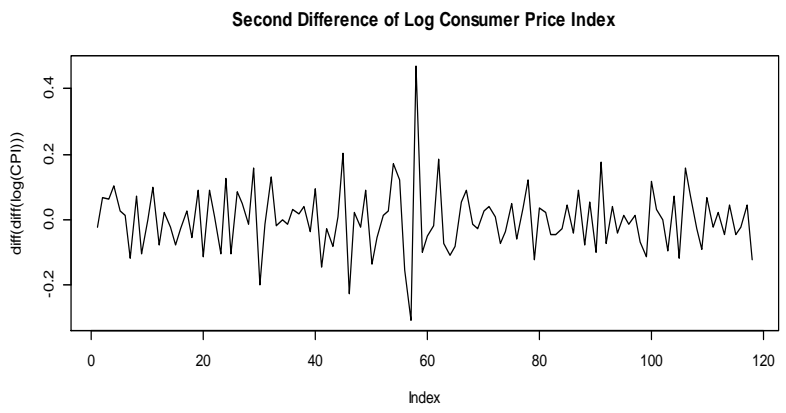




\section{Appendix B}

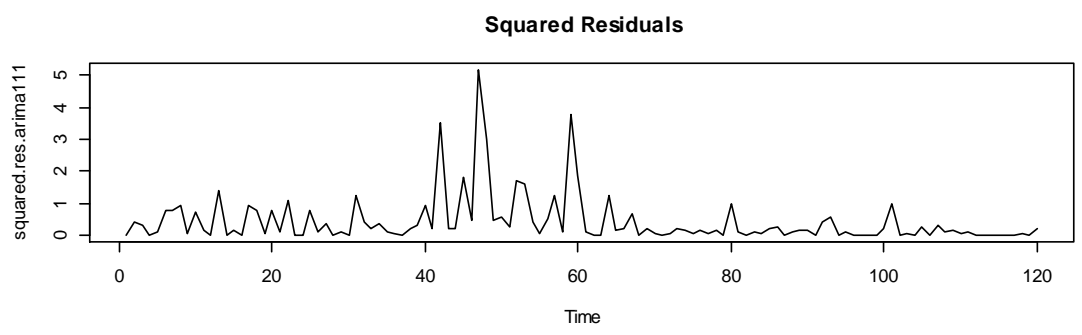

ACF Squared

Residuals

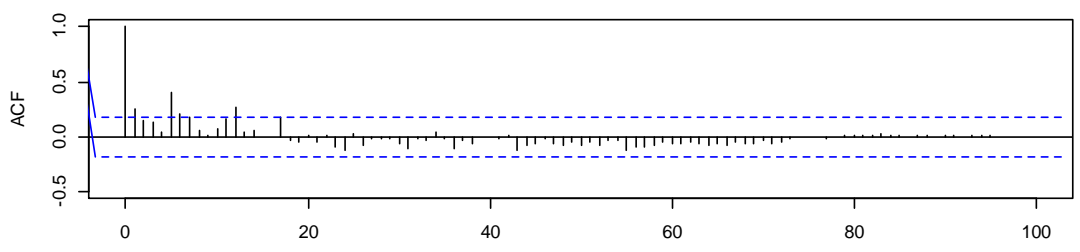

Lag

PACF Squared

Residuals

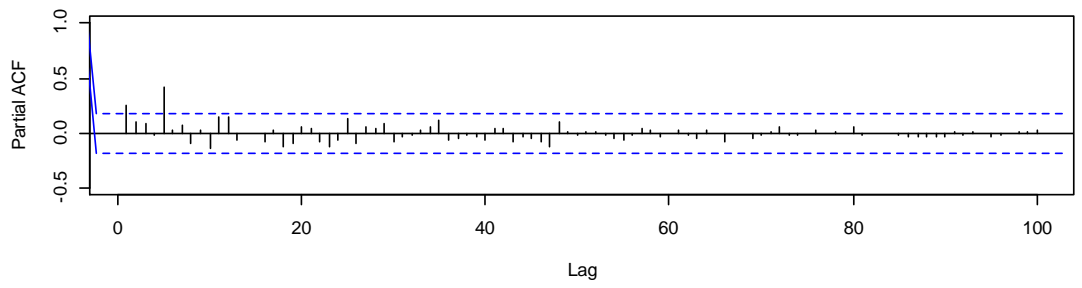

\section{Appendix C}
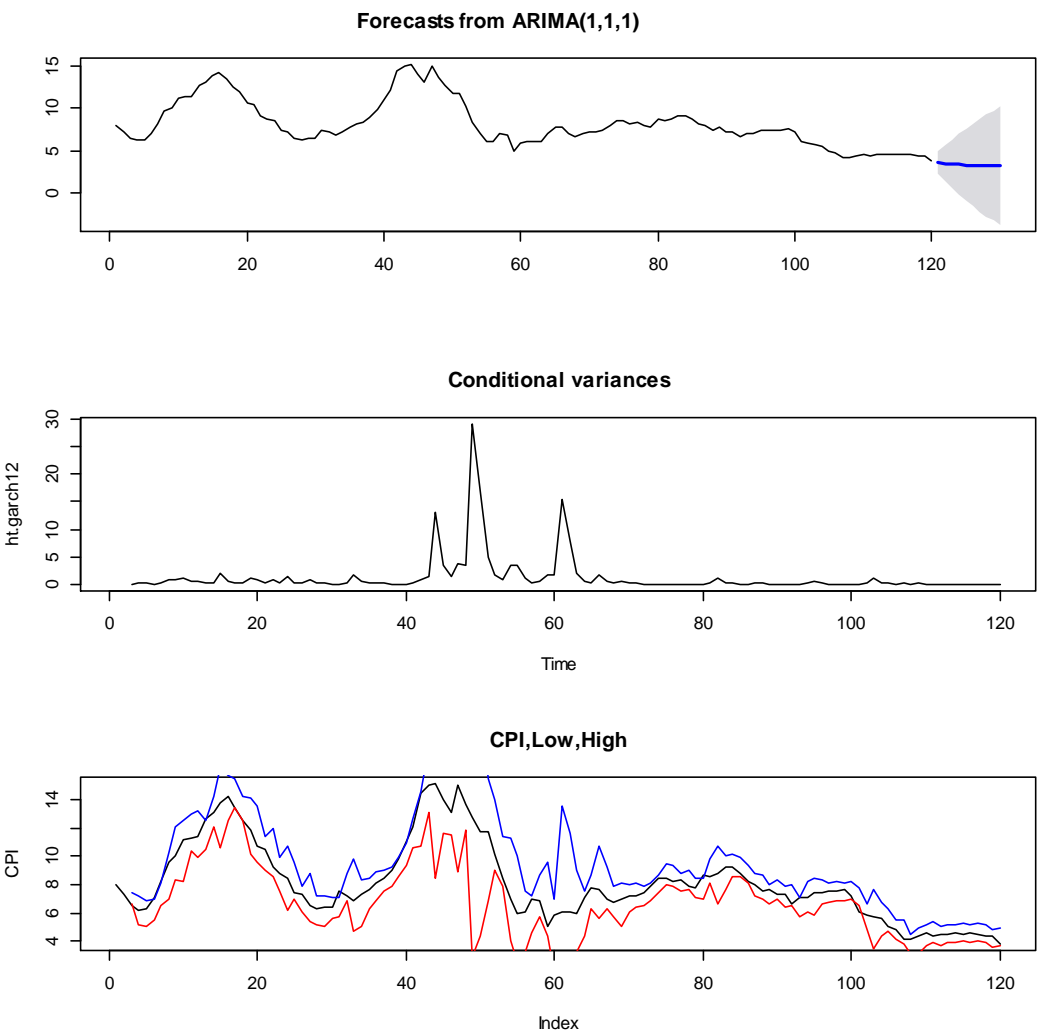


\section{Appendix D}
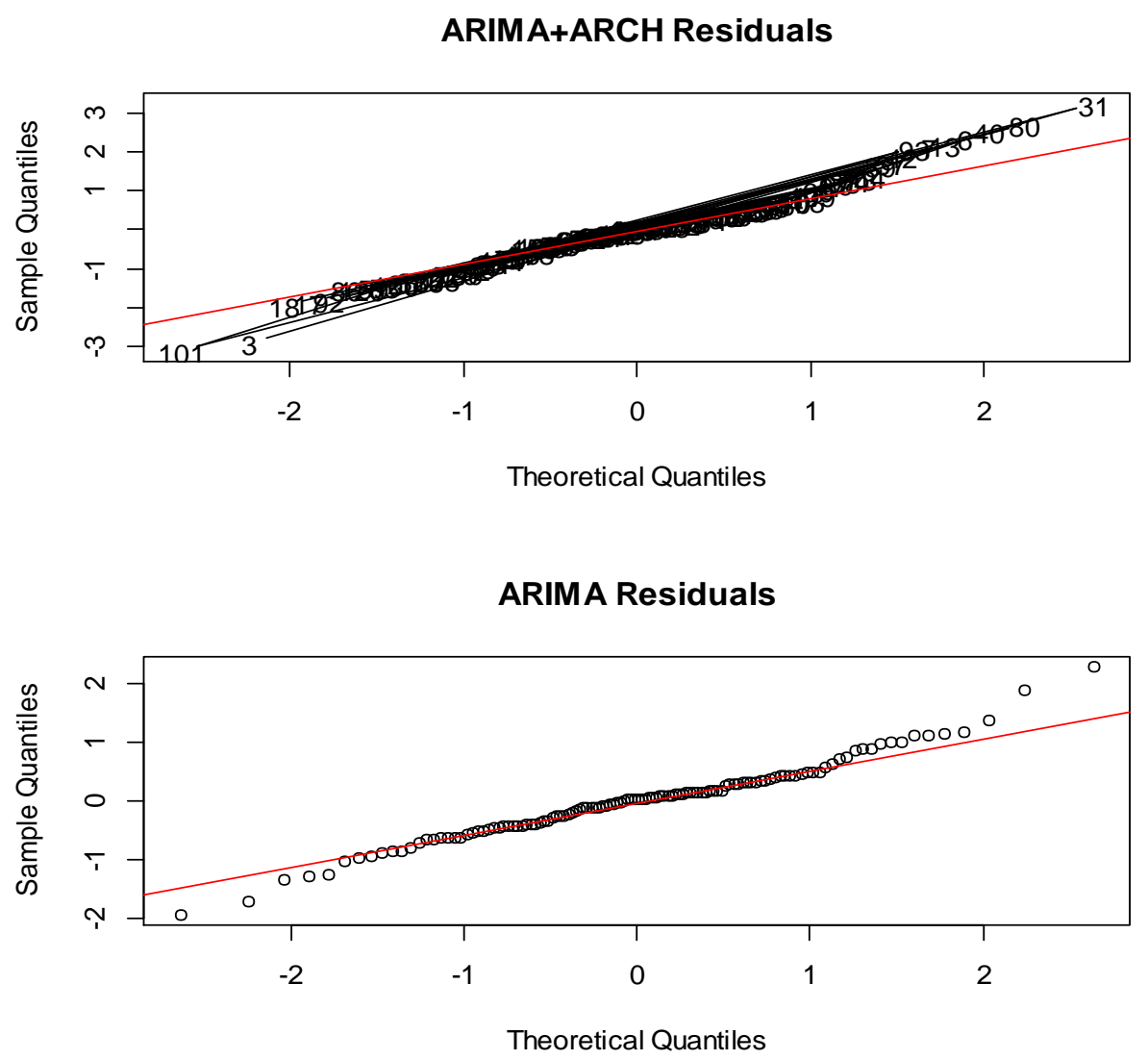

\section{Copyrights}

Copyright for this article is retained by the author(s), with first publication rights granted to the journal.

This is an open-access article distributed under the terms and conditions of the Creative Commons Attribution license (http://creativecommons.org/licenses/by/3.0/). 